\title{
EL REPUBLICANISMO FRENTE AL LIBERALISMO IGUALITARIO
}

REPUBLICANISM AGAINST

EGALITARIAN LIBERALISM

LUCIANO VENEZIA .

Luciano Venezia es Investigador Asistente del Consejo Nacional de Investigaciones Científicas y Técnicas / Universidad Nacional de Quilmes.

E-mail: Ivenezia@unq.edu.ar.

En la literatura filosófica que se interesa por la historia del pensamiento político suelen distinguirse dos escuelas interpretativas $^{1}$. En primer lugar, está el enfoque desarrollado por "profanadores de tumbas» de cuño oxoniense. Los estudiosos que adoptan esta perspectiva analítica se preocupan fundamentalmente por lo que los textos clásicos tienen para aportar al debate teórico contemporáneo. Por esta razón, los intérpretes analíticos consideran que, antes que artefactos culturales históricamente situados, estos textos son vehículos de ideas y argumentos más o menos trasparentes. En todo caso, si los textos canónicos tienen ideas o argumentos dudosos, los filósofos los utilizan para desarrollar teorías originales inspiradas en sus tesis centrales ${ }^{2}$. El enfoque articulado por los «arqueólogos» de origen cantabrigense está asimismo presente en la literatura filosófico-política contemporánea. En este caso, los estudiosos consideran que los textos clásicos son piezas de un debate político históricamente situado. De este modo, los investigado-

\footnotetext{
${ }^{1}$ Rosler (2016: 13-19) retoma la distinción de Derek Parfit entre «profanadores de tumbas» y «arqueólogos» y la articula con el eje Oxford-Cambridge.

2 Kavka (1986) y Simmons (1993) son dos ejemplos conspicuos de este tipo de enfoque. Como indican los títulos, Kavka desarrolla una teoría moral y política hobbesiana, mientras que Simmons elabora una teoría lockeana de la obligación política y sus límites.
} 
res contextualistas consideran que, a fin de comprender su significado de forma cabal, las obras clásicas del pensamiento político -al igual que cualquier otro artefacto cultural- deben ser analizadas en el contexto de las convenciones lingüísticas que informan al contexto ideológico de su propio tiempo ${ }^{3}$.

El último libro de Andrés Rosler, $R a-$ zones Públicas. Seis Conceptos Básicos sobre la República, se posiciona en este debate metodológico de forma original. De acuerdo con la argumentación desarrollada en la Introducción, los enfoques analíticos y contextualistas no son necesariamente antagónicos ${ }^{4}$. De hecho, Rosler considera que las posturas analítica y contextualista son "fácilmente compatibles» (Rosler, 2016: 19). Igualmente, la argumentación desarrollada en el texto lo coloca en una posición más cercana al punto de vista analítico. Aun cuando la impresionante erudición de Rosler es un hecho que salta a la vista de los lectores, la característica distintiva del libro es en realidad la combinación de precisión conceptual, claridad expositiva y potencia argumentativa al servicio de la causa republicana ${ }^{5}$. En efecto, las citas de Cicerón, Tito Livio, Maquiavelo, Montesquieu y Rousseau (y también Shakespeare), entre muchos otros, con la contracara fundamentalmente de Hobbes, están destinadas a desarrollar una teoría política particular -el republicanismo- que a juicio de Rosler es fundamentalmente correcta. Rosler considera que «nos preocupamos por una obra no solamente para poder comprender su significado»; a fin de cuentas, «nos interesa saber si tiene algo que agregar, si puede contribuir a la discusión contemporánea como una alternativa a los paradigmas predominantes en el presente» (Rosler, 2016: 19). Por esta razón, Rosler sostiene que «en última instancia, el atractivo o valoración de un discurso, por político que sea, no puede depender de un contexto o de la historia» sino de si son apropiadas o razonables (Rosler, 2016: 19).

Rosler no tiene reparos en admitir que el republicanismo ha estado históricamente comprometido con no pocas instituciones moralmente repugnantes, incluyendo a la esclavitud, la censura, el imperialismo y la dictadura (Rosler, 2016: 19, 63-64, 240-248, 272-282, 307-308). En este sentido, Rosler propone un retrato del republicanismo "con verrugas y todo» (Rosler, 2016: 19). Igualmente, Rosler argumenta que el vínculo del republicanis-

\footnotetext{
${ }^{3}$ Skinner (2011) es un ejemplo típico de este tipo de enfoque.

${ }^{4}$ Me permito señalar que realizo una observación similar en Venezia (2015: 10 n. 23).

${ }^{5}$ Asimismo, el libro tiene una alta cuota de humor e ironía, aunque -me atrevo a especular- los lectores van a estar divididos acerca de cuán logrado está este aspecto del texto.
} 
mo con estas instituciones es accidental y contingente antes que verdaderamente necesario, además de que el propio discurso republicano tiene las herramientas teóricas y retóricas para desarrollar una visión crítica de su propia historia (Rosler, 2016: 20-29, 161, 248, 305-306, 308).

De acuerdo con Rosler, «república» refiere «a un régimen político cuyo valor primordial es la libertad, que depende de la virtud cívica, gira alrededor del debate, sostiene el imperio de la ley, exige una actitud patriota y es fuertemente antipersonalista» (Rosler, 2016: 28). Sobre la base de esta consideración, Rosler dedica en Razones Públicas sendos capítulos a analizar de forma detallada la libertad, la virtud, el debate, la ley, la patria y César, «el enemigo natural e interno del republicanismo» (Rosler, 2016: 257). Este elemento del libro merece ser destacado. En efecto, una característica distintiva del texto de Rosler es el desarrollo pormenorizado de los diferentes elementos del republicanismo, que muchas veces son tratados de forma superficial o son directamente ignorados incluso en los mejores trabajos de filosofía política analítica contemporánea.

Repasemos brevemente la estructura del libro. Por supuesto, el discurso republicano está asociado a un concepto especifico de libertad como no dominación. Consiguientemente, Rosler dedica un capítulo al análisis detallado de esta noción (capítulo 1). Pero allí no termina la narra- ción histórico-conceptual (Rosler, 2016: 19) desarrollada en el texto. Además de una teoría de la libertad, el republicanismo incluye asimismo una concepción de la virtud cívica (capítulo 2). Asimismo, el debate político entre argumentos igualmente atendibles es un elemento central del discurso republicano (capítulo 3). El reverso de la libertad, la autoridad de la ley, es también una característica fundamental de esta filosofía política (capítulo 4). Finalmente, el libro desarrolla la idea de que el republicanismo exige una actitud patriota de los ciudadanos (capítulo 5). Por otro lado, Rosler destaca que el republicanismo es fuertemente antipersonalista y por tanto dedica un capítulo a César y el cesarismo (capítulo 6).

Rosler desarrolla un análisis matizado de estos diferentes conceptos en los seis capítulos que conforman Razones Públicas. Un ejemplo en este sentido es el tratamiento de la noción de ley desarrollado en el capítulo 4. La comparación con Republicanismo. Una teoría sobre la libertad y el gobierno de Philip Pettit quizá sea ilustrativa. Aun cuando no propone un análisis pormenorizado, especialmente en la primera parte de su libro, Pettit asume que la ley es esencialmente coercitiva. De hecho, Pettit apela a esta concepción para argumentar en contra del liberalismo y a favor del republicanismo (Pettit, 1999, primera parte). Por su parte, Rosler muestra que las cosas son en realidad más 
complicadas. En primer lugar, Rosler distingue dos concepciones de la autoridad: minimalista y maximalista (Rosler, 2016: 176-178, 180-183). La autoridad minimalista no modifica sustancialmente el razonamiento práctico; a lo sumo nos recuerda lo que debemos hacer o nos castiga en caso de incumplimiento. En cambio, la autoridad maximalista es esencialmente práctica; en definitiva, las directivas de una autoridad maximalista introducen obligaciones morales. Acá Rosler se basa en el análisis de la autoridad de Joseph Raz: «[e]l hecho de que una autoridad requiera la realización de una acción es una razón para su realización que no se ha de añadir a las otras razones relevantes cuando se evalúa qué hacer, sino que debe excluir y tomar el lugar de alunas de ellas» (Raz, 1990: 124, cit. en Rosler, 2016: 180, énfasis removido). Sobre la base de esta caracterización, Rosler muestra que la autoridad republicana es efectivamente maximalista. En efecto, la autoridad republicana tiene por objetivo resolver desacuerdos genuinos entre ciudadanos republicanos cuya motivación y virtud son insospechables (Rosler, 2016: 183). A su vez, el análisis de la autoridad del derecho muestra la estrecha conexión conceptual entre los diferentes elementos del discurso republicano. La virtud no impide sino que estimula el debate político, mientras que la ley es el reverso de la libertad y la patria es el espacio apropiado para que los ciudadanos vivan en libertad y puedan debatir en el marco del Imperio de la Ley (Rosler, 2016: 29).

Tal como señalé recién, Rosler argumenta en Razones Públicas que la libertad, la virtud, el debate, la ley, la patria son los elementos característicos del republicanismo. Al mismo tiempo Rosler afirma que la libertad como no dominación constituye la noción sustantiva central del republicanismo: «[t]odo el discurso republicano gira alrededor de la libertad» (Rosler, 2016: 29; ver también 113, 161, 305 y 308). Por esta razón, ahora voy a centrar mi atención sobre la forma en que Rosler defiende al republicanismo por medio de su caracterización del concepto de libertad. El foco de mi análisis no es la descripción de la libertad republicana desarrollada en el texto. De hecho, la presentación y evaluación crítica de las concepciones negativa y positiva y la consiguiente defensa de la versión republicana de la libertad desarrollada en el primer capítulo de Razones Públicas es excelente y tranquilamente puede utilizarse como una verdadera introducción a la discusión filosófico-política contemporánea sobre esta cuestión. A mi juicio, en cambio, sí es problemática la contraposición del republicanismo frente a otras teorías normativas alternativas desarrollada en el libro.

Aun cuando la mayor parte del texto está destinado a desarrollar las características del republicanismo clásico mencio- 
nadas en la sección 1, la argumentación de Rosler tiene asimismo una dimensión polémica. En efecto, una tesis central de Razones Públicas es que el republicanismo es una concepción filosófico-política superadora frente a la alternativa de los dos discursos políticos -liberalismo y populismo- que informan tanto el debate ideológico como la práctica política contemporánea (Rosler, 2016: 308). Por mi parte, no tengo mayores dudas respecto del populismo, pero no estoy tan seguro del resultado de la batalla frente al liberalismo, fundamentalmente al liberalismo igualitario, al menos sobre la base de las consideraciones desarrollas en el libro.

El punto de partida del argumento de Rosler es la distinción de Isaiah Berlin entre libertad negativa y libertad positiva (Berlin, 2005). En concreto, Rosler describe estas dos versiones del concepto de libertad (Rosler, 2016: 33-52) y luego desarrolla y defiende la concepción republicana (Rosler, 2016: 53-66). Por supuesto, Rosler sostiene que la teoría de la libertad republicana en términos de no dominación es superadora; de hecho, considera que la distinción berliniana entre los conceptos negativo y positivo de libertad está hecha a la medida del republicanismo (Rosler, 2016: 33). Esta tesis es sin dudas atendible; efectivamente, las versiones de la libertad como ausencia de interferencia y como autodominio o autocontrol no agotan el espacio lógico y por consiguiente, dejan lugar para una formulación de la libertad que combina elementos de estos dos aspectos de la libertad, es decir, la libertad como no dominación republicana. Así y todo, esta idea no conlleva -como sugiere el argumento desarrollado en Razones Públicas- que el republicanismo es por consiguiente una teoría política superadora.

Rosler afirma que el valor o ideal político de la libertad es aceptado prácticamente por todas las posiciones ideológicas que informan el debate teórico contemporáneo (Rosler, 2016: 31-32 y 46). Aun cuando esta consideración es pertinente, al mismo tiempo no implica que toda la discusión y argumentación filosóficopolítica deba ser desarrollada en estos términos. Por ejemplo, esta posición conlleva que la justicia debe estar únicamente determinada por la libertad, razón por la cual desarrollar un análisis adecuado del concepto de libertad se vuelve una tarea fundamental para articular y defender una concepción sustantiva de la justicia distributiva. Sin embargo, esta forma de plantear las cosas no parece realmente adecuada y en todo caso no describe la manera de proceder de los defensores contemporáneos del liberalismo, especialmente del liberalismo igualitario.

Entre las diferentes versiones contemporáneas del liberalismo, sólo el libertarianismo à la Robert Nozick considera que la libertad negativa es el único valor político. En particular, Nozick sostie- 
ne que únicamente su teoría intitular es compatible con la libertad de las personas, lo que le permite sostener que, por esa razón, sólo esta concepción de la justicia distributiva está moralmente justificada. ${ }^{6}$ Ahora bien, no es totalmente claro que el libertarianismo nozickeano sea en verdad una teoría política liberal. ${ }^{7}$ En cambio, los liberales de corte igualitario consideran que, además de la liberad como no interferencia, hay asimismo otros valores que deben informar las principales instituciones de una sociedad justa, incluyendo por supuesto a la igualdad. Por ejemplo, los principios de justicia presentados y defendidos por John Rawls en Teoría de la justicia y otros textos caracterizan una concepción de la justicia distributiva que, según su autor, incorpora los ideales de libertad e igualdad y, también, fraternidad. ${ }^{8}$

Asimismo, algunos filósofos liberales, incluyendo al propio Rawls, distinguen entre el significado de la libertad y el valor de la libertad ${ }^{9}$. El punto de la distinción, precisamente, es que el mero análisis del concepto de libertad en términos de ausencia de interferencia no determina una posición normativa adecuada. De este modo, en la teoría de la justicia rawlsiana la libertad negativa de las personas está sujeta a restricciones sobre la base de la idea de igual libertad y del orden lexicográfico de los dos principios de justicia. Junto con Pettit, por ejemplo, Rosler podría señalar que este tipo de maniobras argumentativas precisamente muestra los defectos de la posición de Rawls, mientras que el republicanismo está en condiciones de realizar todo el trabajo argumentativo con su concepción de la libertad como no dominación (Pettit, 1999) ${ }^{10}$. Sin embargo, no es realmente claro que este hecho sea un defecto del liberalismo igualitario; a lo sumo, se trata de un problema de simplicidad argumentativa antes que una dificultad normativa ${ }^{11}$.

\footnotetext{
${ }^{6}$ Nozick (1988: especialmente 154-167). Ver Hevia y Spector (2008) para una presentación y análisis crítico del argumento de Nozick.

7 Ver Freeman (2001) para un argumento en este sentido.

8 Ver Rawls (1995: esp. § 17).

${ }^{9}$ Rawls (1995: § 32).

${ }^{10}$ Dado que no es el objeto de este trabajo, en este contexto meramente asumo -lo que sin dudas es una gran suposición- que la teoría de la justicia distributiva que se sigue del republicanismo es moralmente adecuada.

${ }^{11}$ Por cierto, Pettit considera que la cuestión no es meramente de simplicidad argumentativa. En cambio, sostiene que mientras nociones como la libertad son aceptadas por la mayor parte de las corrientes teóricas e ideológicas, la igualdad es un valor o ideal político mucho más controvertido (Pettit, 1999: 30). Rosler no abunda sobre esta cuestión, por lo que la lectura del texto no permite determinar si comparte este razonamiento. De cualquier forma, creo que el punto de Pettit es discutible. En efecto,
} 
Razones Públicas es un libro excelente. en este sentido no permiten mostrar la La caracterización de Rosler de los rasgos superioridad del republicanismo sobre el fundamentales del republicanismo es, a la vez, erudita y analítica, tal como ilustra la presentación de la discusión de Rosler del concepto de Ley que desarrollamos primeramente.

$\mathrm{Al}$ mismo tiempo, el ensayo de Rosler tiene una dimensión polémica. En efecto, Rosler no sólo realiza un análisis de los elementos característicos del discurso republicano clásico. Además, presenta al republicanismo como una alternativa al populismo y al liberalismo. Por nuestra parte, argumentamos que las consideraciones desarrolladas en Razones Públicas

Los comentarios críticos realizados no ponen en cuestión el valor de las ideas desarrolladas en Razones Públicas; antes bien, la asumen como punto de partida. En efecto, el libro de Andrés Rosler no sólo es un texto erudito; es, también, un ensayo polémico, que no va a resultar indiferente a sus lectores. En este sentido, la discusión que desarrollamos este trabajo es, antes que nada, un reconocimiento a la obra de un escritor admirable, verdadera rara avis en la academia argentina de nuestros días.

filósofos como Ronald Dworkin arguyen que la igualdad -entendida de una manera particularmente abstracta en términos de igual consideración y respeto- es el valor fundamental del liberalismo qua posición política general en oposición al conservadurismo. Ver, por ejemplo: Dworkin (1983: esp. 150-158). Asumiendo la pertinencia de este señalamiento, Will Kymlicka toma a la igualdad como punto de partida en su presentación de las diferentes teorías de la justicia que forman parte del debate filosófico-político contemporáneo, incluyendo al libertarianismo y al liberalismo igualitario, pero también al republicanismo cívico, entre otras. Ver Kymlicka (2002: 3-4). 


\section{Bibliografía}

BERLIN, ISAIAH (2005): «Dos conceptos de libertad», en: Isaiah Berlin, Dos conceptos de libertad y otros escritos, Ángel Rivero (trad.), Madrid, Alianza.

DWORKIN, RONALD (1983): «Liberalismo», en: Stuart Hampshire (comp.), Moral pública y privada, Mercedes Córdoba (trad.), México, Fondo de Cultura Económica.

FREEMAN, SAMUEL (2001): «llliberal Libertarians: Why Libertarianism Is Not a Liberal View», en: Philosophy and Public Affairs, 30(2): 105-151. HEVIA, MARTíN Y SPECTOR, EZEQUIEL (2008): «The Bizarre World of Historical Theories of Justice: Revisiting Nozick's Argument», en: Social Theory and Practice, 34(4): 533-549.

KAVKA, GREGORYS. (1986): Hobbesian Moral and Political Theory, Princeton, Princeton University Press. KYMLICKA, WILL (2002): Contemporary Political Philosophy: An Introduction, segunda edición, Oxford, Oxford University Press.

NOZICK, ROBERT (1998): Anarquía, Estado y utopía, Rolando Tamayo (trad.), Buenos Aires, Fondo de Cultura Económica.

\section{Registro bibliográfico}

VENEZIA, LUCIANO

«El republicanismo frente al liberalismo igualitario», en: ESTUDIOS SOCIALES, revista universitaria semestral, año XXVII, $n^{\circ}$ 52, Santa Fe, Argentina, Universidad Nacional del Litoral, enero-junio, 2017, pp. 161-168.
PETTIT, PHILIP (1999): Republicanismo. Una teoría sobre la libertad y el gobierno, Antoni Domènech (trad.), Barcelona, Paidós.

RAWLS, JOHN (1995): Teoría de la justicia, María Dolores González (trad.), México, Fondo de Cultura Económica.

RAZ, JOSEPH (1990): «Authority and Justification», en: Joseph Raz (comp.), Authority, Nueva York, New York University Press.

ROSLER, ANDRÉS (2016): Razones Públicas. Seis Conceptos Básicos sobre la República, Buenos Aires, Katz Editores.

SIMMONS, A. JOHN (1993): On the Edge of Anarchy: Locke, Consent, and the Limits of Society, Princeton, Princeton University Press.

SKINNER, QUENTIN (2011): Hobbes y la libertad republicana, Juliana Udi (trad.), Bernal, Editorial de la Universidad Nacional de Quilmes - Prometeo 3010.

VENEZIA, LUCIANO (2015): Hobbes on Legal Authority and Political Obligation, Basingstoke, Palgrave Macmillan.

\section{Descriptores - Describers}

Andrés Rosler / Republicanismo / Liberalismo

Andrés Rosler / Republicanism/ Liberalism

Recibido: 26 / 09 / 2016

Aprobado: 14 / 03 / 2017 